\title{
Raman spectroscopy of hypersonic shock waves
}

\author{
A. Ramos, B. Maté, G. Tejeda, J. M. Fernández, and S. Montero* \\ Instituto de Estructura de la Materia, Consejo Superior de Investigaciones Científicas, Serrano 123, 28006 Madrid, Spain
}

(Received 1 March 2000; revised manuscript received 25 May 2000)

\begin{abstract}
Raman spectroscopy is shown to be an efficient diagnostic methodology for the study of hypersonic shock waves. As a test, absolute density and rotational population profiles have been measured across five representative normal shock waves of $\mathrm{N}_{2}$ generated in a free jet, spanning the Mach number range $7.7<M<15.3$. The interconversion of three differentiated populations (cold, scattered, and rethermalized molecules) across these shock waves shows a largely bimodal rotational distribution function with additional contribution of scattered molecules, in close analogy with the velocity distribution function known from helium shock waves [G. Pham-Van-Diep et al., Science 245, 624 (1989)]. Quantitative data on invariance trends of density profiles and properties of the wake beyond the shock waves are reported.
\end{abstract}

PACS number(s): 47.40.Nm, 47.80.+v, 33.20.Fb,

\section{INTRODUCTION}

The practical implications and the conceptual difficulties of shock waves have attracted the attention of engineers and scientists for a long time. Gas shock waves are generated in various natural and artificial processes where the medium is subject to strong pressure, thermal, density, and velocity gradients, with breakdown of thermodynamic equilibrium. Though the general properties of such a perturbed medium are well established, the behavior of the distribution function across a shock wave is poorly known, specially in molecular gases where rotational and vibrational degrees of freedom complicate the solution of Boltzmann equation governing its evolution in space and time. The solution proposed by MottSmith [1] accounts for a number of properties of onedimensional (1D) shock waves in monatomic gases. 2D and $3 \mathrm{D}$ shock waves are a challenge not only for theoreticians but also for experimentalists.

In contrast to the abundant theoretical literature on shock waves [1-4], experimental studies capable to provide information at microscopic scale are scarce. These are largely based on electron beam induced fluorescence [5-7], and electron beam absorption [8], in combination with shock tubes, free jets, and wind tunnels. These studies have confirmed in part the main conclusions of Mott-Smith (MS) theory [1].

In the present work we show the potential of high sensitivity Raman spectroscopy, based on CCD detection, to investigate shock waves experimentally at molecular scale, with advantage over current alternative methods. As has been shown recently [9], Raman spectroscopy features some unique capabilities to probe molecular gas media under the strong nonequilibrium conditions prevailing in shock waves. Its more outstanding merits are (a) universality, in the sense that many relevant molecular species $\left(\mathrm{N}_{2}, \mathrm{O}_{2}, \mathrm{H}_{2}, \mathrm{CO}\right.$, $\mathrm{N}_{2} \mathrm{O}, \mathrm{H}_{2} \mathrm{O}, \mathrm{CO}_{2}, \mathrm{NH}_{3}$, light hydrocarbons, etc.) are easily detectable, (b) spatial resolution of few $\mu \mathrm{m}$, (c) linear relation between measured spectral intensities and local molecular densities, (d) dynamical range of intensities ( $\propto$ to densities)

*Email address: emsalvador@iem.cfmac.csic.es of over six orders of magnitude, (e) capacity to measure accurately local rotational populations, and (f) long term stability of several hours. These merits adapt well to the detailed investigation of shock waves in molecular gases, in particular of their strong density and thermal gradients occuring at scale of a few mean free paths [8].

\section{EXPERIMENTAL SETUP}

The equipment used here has been devised as a miniature facility for the study of supersonic flows in a wide range of Mach numbers, with diagnostics based on Raman spectroscopy. It is composed of an expansion chamber to generate stationary supersonic jets and associated shock waves $[9,10]$, and a very high sensitivity spectrometer to record Raman spectra at selected points within the jet [11].

\section{A. Expansion chamber}

The expansion chamber, of $42 \times 42 \times 30 \mathrm{~cm}^{3}$, manufactured in aluminum, contains the jet nozzle, the optical (multipass) system to excite the Raman scattering, part of the optical system to collect the Raman signal, and the associated micropositionig devices for nozzle and optical elements. For a better optomechanical stability these parts are firmly attached to the $4 \mathrm{~cm}$ thick basis of the chamber. All ten micropositioning degrees of freedom internal to the chamber (three for the nozzle, three for the laser beam focusing lens, three for the multipass mirror, and one for the collection optics) are operated by remote control from outside the chamber. A more detailed description may be found in Ref. [9].

A scheme of the Raman scattering excitation optics is shown in Fig. 1. The Raman signal is collected along $y$-axis by means of an $f: 0.95$ composed optical system of magnifying power $\times 10$. A 2080 Spectra Physics $\mathrm{Ar}^{+}$laser source, with highly improved beam pointing and output power stability, was used for excitation. Typical excitation power was $6 \mathrm{~W}$ at $\lambda=514.5 \mathrm{~nm}$. For the focusing lens of $f=35 \mathrm{~mm}$ focal lenght the laser beam waist is $14 \mu \mathrm{m}$. The multipass system increases this figure to about $20 \mu \mathrm{m}$, with a signal gain factor $\times 5$. 


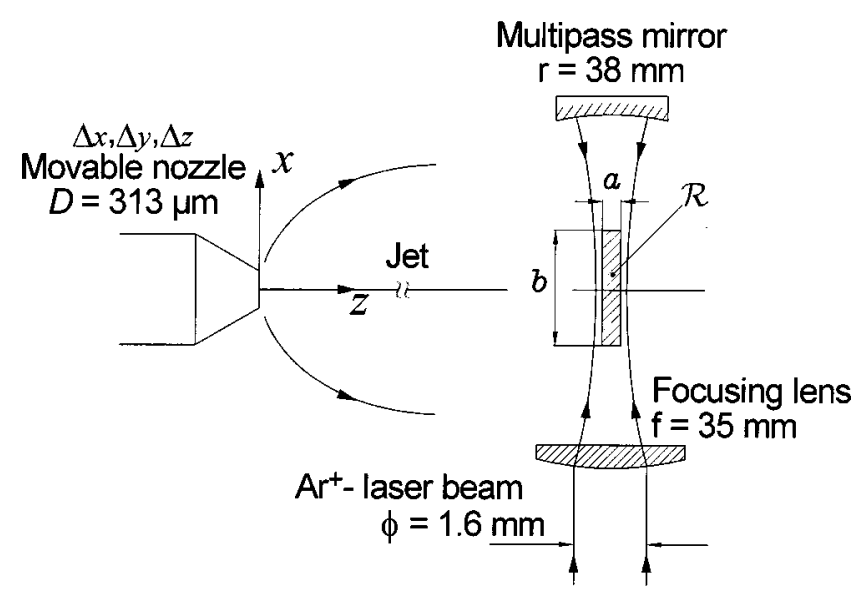

FIG. 1. Scheme of the Raman scattering excitation optics for the investigation of supersonic jets.

The expansion chamber operates in stationary flow regime. It is continuously evacuated by means of a Roots pump of $1430 \mathrm{~m}^{3} / \mathrm{h}$, backed by a rotary pump of $70 \mathrm{~m}^{3} / \mathrm{h}$, while the sample gas is injected at stagnation pressure $P_{0}$ through the nozzle. Due to the residual pressure $P_{r}$ in the expansion chamber, a stationary shock wave associated to the supersonic jet is formed at distance [12]

$$
z_{M}=0.67 D\left(P_{0} / P_{r}\right)^{1 / 2}
$$

from the nozzle of diameter $D$. Within certain limits $P_{r}$ can be accurately controlled at will by means of an auxiliary gas inlet into the expansion chamber. The position of the shock waves discussed below has been fixed this way.

\section{B. Spectrometer}

The very high sensitivity Raman spectrometer, a noncommercial instrument commissioned in our laboratory [11], is equipped with a 2360 lines/mm holographic grating of $102 \times 102 \mathrm{~mm}^{2}$ as dispersive element, and a $512 \times 512$ pixel CCD detector refrigerated by liquid nitrogen. Scanning and data acquisition are computer controlled. For a laser excitation power of $6 \mathrm{~W}$ the routine detection limit is on the order of $10^{19}$ molecules $/ \mathrm{m}^{3}$ at a spectral resolution of $1 \mathrm{~cm}^{-1}$, a detection power on the order of $1 \mathrm{ppm}$ referred to a gas sample at standard conditions of pressure and temperature. This figure is between two and three orders of magnitude better than for any commercially available instrument.

\section{INVESTIGATED SHOCK WAVES}

In order to illustrate the latent capacity of Raman spectroscopy for the quantitative study of hypersonic shock waves, the density and rotational temperature profiles of five reference shock waves have been measured extensively. They were generated by the expansion of $\mathrm{N}_{2}$ through a nozzle of diameter $D=313 \mu \mathrm{m}$, and internal length $L$ $=1 \mathrm{~mm}$, under nominal constant stagnation pressure $P_{0}$ $=100 \mathrm{kPa}$ and temperature $T_{0}=295 \mathrm{~K}$. These five shock waves, henceforth referred to as A, B, C, D, and E, are located at distances of about 9, 18, 27, 36, and $45 \mathrm{D}$ from the nozzle, and span the Mach number range $7.7 \leqslant M \leqslant 15.3$, according to the usual estimate $[10,13]$. The locations were

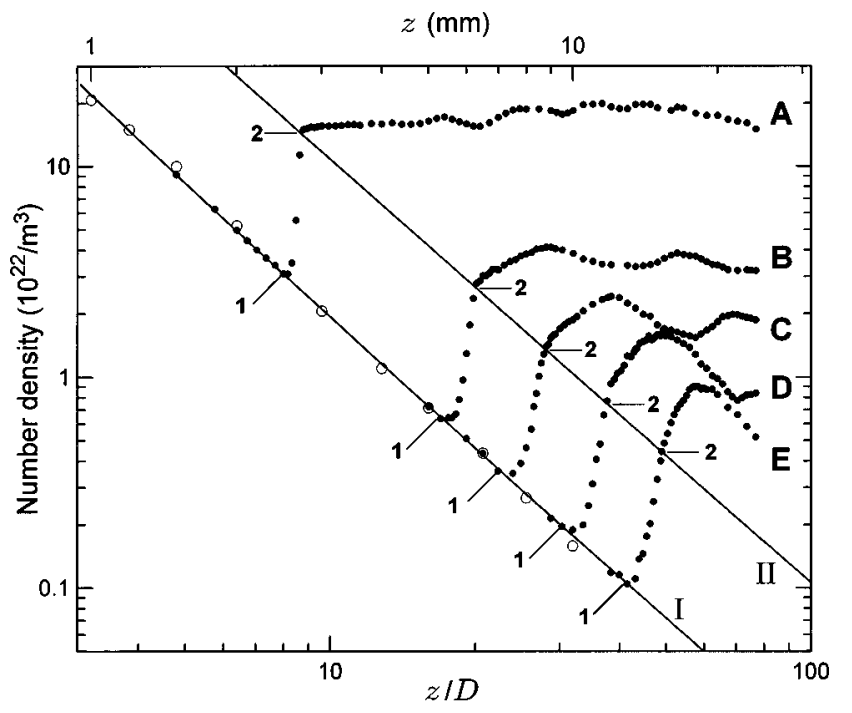

FIG. 2. Absolute number densities of $\mathrm{N}_{2}$ shock waves along the expansion axis as measured by Raman spectroscopy.

fixed by the ratios of stagnation to residual pressure $P_{0} / P_{r}$, for $P_{r}=420,100,50,28$, and $18 \mathrm{~Pa}$, respectively, allowing some atmospheric air to enter the expansion chamber through the auxiliary needle valve.

The density and temperature profiles of shock waves A, B, C, D, and E, are shown in Figs. 2 and 3. For $P_{0}$ $=100 \mathrm{kPa}$ and $P_{r}<5 \mathrm{~Pa}$ no shock wave is produced in the investigated region of the jet $(z<25 \mathrm{~mm})$, the density decaying along the expansion as $z^{-2}$ within experimental accuracy, as shown by the white circles in Fig. 2. Continuous line I represents an isentropic expansion of an ideal gas with a heat capacity ratio $\gamma=7 / 5$ fitted to the experimental data points. This fit yields an effective stagnation pressure $P_{0}^{\text {eff }}$ $=80 \mathrm{kPa}, 20 \%$ smaller than the nominal one, $P_{0}=100 \mathrm{kPa}$, measured by means of a precision gage pressure transducer located in the $\mathrm{N}_{2}$ feeding line, upstream from the nozzle. This reduction reflects the departure from ideal nozzle conditions caused by geometry, gas viscosity, and boundary layer.

\section{MEASURING PROCEDURE}

The region actually "seen" by the spectrometer is denoted by $\mathcal{R}$ in Fig. 1. It determines the spatial resolution of the experiment. Dimension $a$ of $\mathcal{R}$ is controlled by means of the entrance slitwidth of the spectrometer; dimension $b$, by the vertical binning system of the CCD detector. Several hundred Raman spectra were recorded at slitwidth ranging from 100 to $150 \mu \mathrm{m}$, and CCD vertical binning from 150 to 300 pixel, depending on the particular shock wave. These conditions correspond to an effective spatial resolution 10 $<a<15 \mu \mathrm{m}$, and $650<b<1300 \mu \mathrm{m}$, the spectral resolution ranging from 1.3 to $1.9 \mathrm{~cm}^{-1}$. In all cases dimension $a$ was at least nine times narrower than the shock wave thickness $\delta$, and dimension $b$, less than one third of the diameter of the planar section of the shock wave. Distance $z$ between nozzle and observed region $\mathcal{R}$ is controlled by a codified digital micropositioner with accuracy of $\pm 1 \mu \mathrm{m}$. Errors in the $z / D$ abscissas of data points in Figs. 2 and 3 are therefore negligible. 


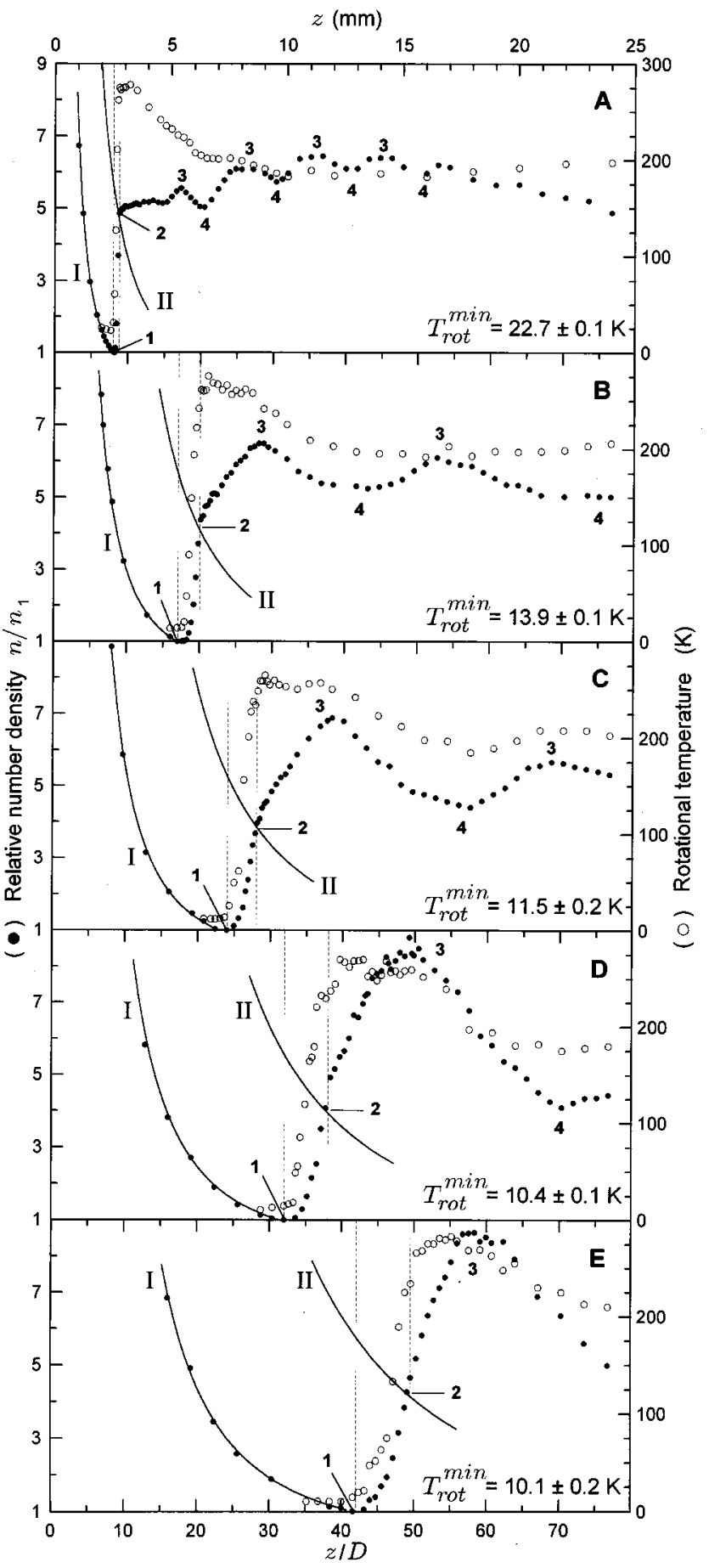

FIG. 3. Relative number densities (-), and rotational temperatures $(\mathrm{O})$ of $\mathrm{N}_{2}$ shock waves along the expansion axis. Vertical dashed lines indicate the limits of the shock waves.

As shown in Fig. 2 the number density of the medium investigated here is in the range $10^{21}<n<10^{23} \mathrm{~m}^{-3}$, i.e., between two and four orders of magnitude below that of a gas at standard conditions. Nonetheless the peak signal of the spectral lines is on the order of ten CCD counts per second (cps), while the noise is about $0.1 \mathrm{cps}$ for a data acquisition period [11] of 20 seconds. With a signal to noise ratio about 100 the recorded Raman spectra-one per reported data point-are good enough to produce high quality rotational temperature $( \pm 5 \%)$, and absolute density data $( \pm 10 \%)$.

\section{A. Densities}

To a very good approximation the integrated Raman intensity of the $Q$ branch of the vibrational band of $\mathrm{N}_{2}$ at 2331 $\mathrm{cm}^{-1}$,

$$
I_{2331}=K \times n,
$$

is proportional to the number density $n$ of the observed medium. $K$ depends on exciting irradiance and wavelenght, scattering geometry, and molecular polarizability derivative of $\mathrm{N}_{2}$, factors which remain constant along the experiment, and on the local temperature of the gas. For temperature below $300 \mathrm{~K}$ this dependence is neglegible. On this basis Raman spectroscopy provides by means of Eq. (2) the most reliable procedure to measure the number density of gas media with strong gradients in a range of densities of at least six orders of magnitude. Moreover, relative number densities can be converted to absolute values by comparison with a reference medium of known number density, in present case, the same gas sample under static conditions.

The density profiles reported in Figs. 2 and 3 were measured as described above as a function of the reduced distance $z / D$ from the nozzle. Absolute calibration was carried out comparing the Raman signal at selected points of the expansions with the corresponding signal from static $\mathrm{N}_{2}$ at $P=20 \mathrm{kPa}$ and $T=295 \mathrm{~K}$. In order to emphasize specific features of shock waves, the absolute density profiles are plotted in Fig. 2 in a double-logarithmic scale, while the relative densities normalized to the minimum are plotted in Fig. 3 referred to a linear scale, jointly with the rotational temperature.

\section{B. Population of rotational levels}

The intensities of the rotational lines associated to $J \rightarrow J$ +2 Raman transitions of linear molecules may be expressed as

$$
I_{J \rightarrow J+2}=G \times P_{J} \times \frac{(J+1)(J+2)}{(2 J+1)(2 J+3)} .
$$

Factor $G$ depends on exciting irradiance and wavelenght, scattering geometry, and anisotropy of the molecular polarizability, and remains constant along the experiment. $P_{J}$ is the fractional population of rotational level of quantum number $J$, with $\Sigma_{J} P_{J}=1$. Present $P_{J}$ 's were measured as a function of $z / D$ from the $I_{J \rightarrow J+2}$ intensities of the rotational spectra of $\mathrm{N}_{2}$ spanning the range between 5 and $180 \mathrm{~cm}^{-1}$.

It must be emphasized that Eq. (3) is strictly valid regardless of whether the gas sample is in thermodynamical equilibrium or not. Indeed, the interior of the shock waves, between points 1 and 2 of Figs. 2 and 3, is characterized by a pronounced breakdown of Boltzmann distribution of population.

\section{Rotational temperatures}

Thermodynamical equilibrium of the rotational levels is characterized by a Boltzmann distribution of population

$$
P_{J}=\left(Z_{\mathrm{rot}}\right)^{-1} g_{J}(2 J+1) e^{-\beta B J(J+1) / T_{\text {rot }}},
$$



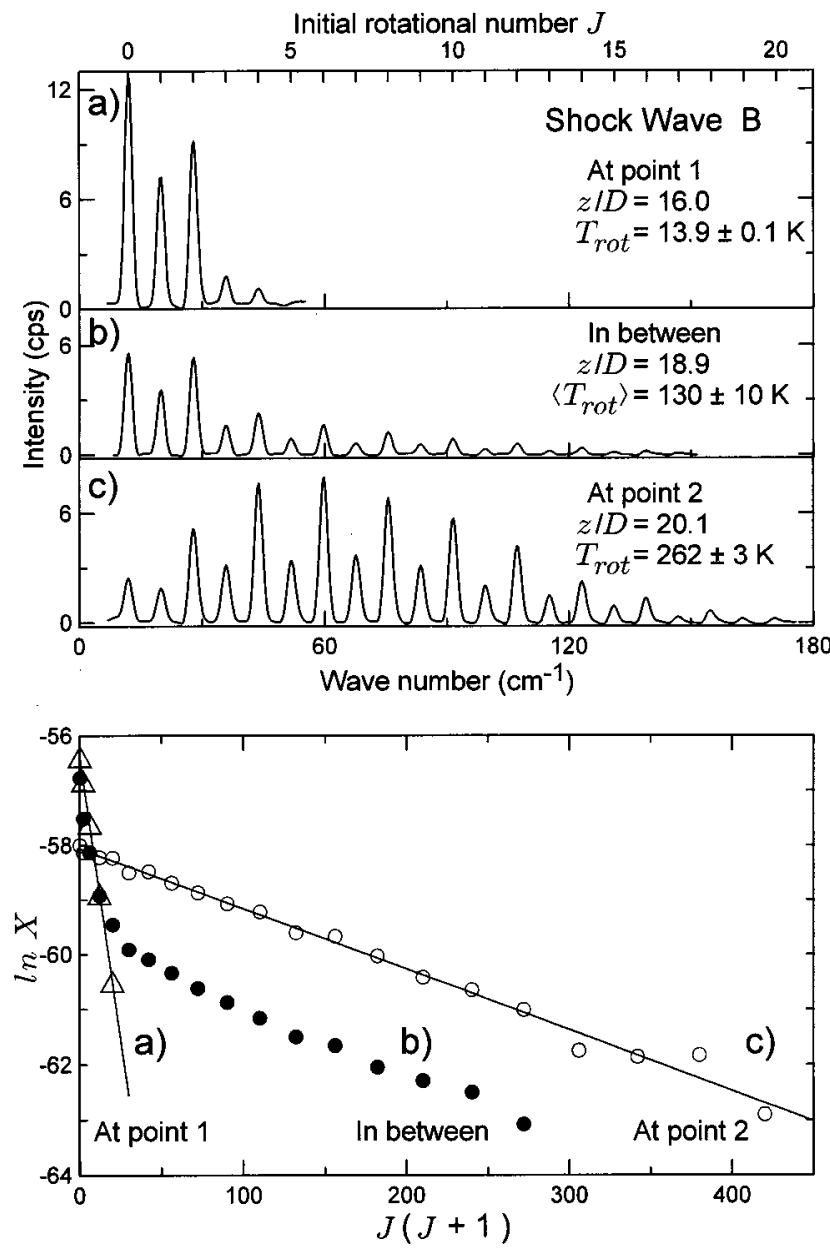

FIG. 4. Top: rotational Raman spectra of $\mathrm{N}_{2}$ recorded (a) before, (b) within, and (c) after the shock wave B. Bottom: their respective Boltzmann plots; $X=P_{J} /\left[g_{J}(2 J+1)\right]$.

where $Z_{\text {rot }}$ is the rotational partition function, $g_{J}$ the spin statistical weight, $\beta=h c / k=1.4388 \mathrm{~cm} \mathrm{~K}, \quad B=1.98973$ $\mathrm{cm}^{-1}$ the rotational constant of $\mathrm{N}_{2}$, and $T_{\text {rot }}$ the rotational temperature, by definition.

Outside from the shock wave, upstream from point 1 , or downstream from point 2 in Figs. 2 and 3, the graph of the function $\ln \left\{P_{J} /\left[g_{J}(2 J+1)\right]\right\}$ versus $J(J+1)$ yields a linear Boltzmann plot. In these two regions the rotational temperatures of Fig. 3 were obtained from the slope of such plots. The lowest temperature attained in each expansion is indicated in Fig. 3 as $T_{\text {rot }}^{\min }$. An example of linear Boltzmann plots from shock wave B is shown in Fig. 4 (bottom), plots (a) and (c).

Conversely, within the shock wave, between points 1 and 2 in Figs. 2 and 3, the Boltzmann plots are nonlinear, as shown in case (b) of Fig. 4. In this case the rotational temperature cannot be defined according to Eq. (4). In order to help visualizing the thermal behavior within the shock wave, the alternative definition of rotational temperature for linear molecules

$$
\left\langle T_{\text {rot }}\right\rangle=\beta \sum_{J} P_{J} B J(J+1),
$$

has been used instead. $\left\langle T_{\text {rot }}\right\rangle$ represents the average rotational energy expressed in units of $\mathrm{K}$. Under equilibrium conditions $\left\langle T_{\text {rot }}\right\rangle$ is strictly equivalent to the definition derived from Eq. (4). Temperatures reported in Fig. 3 in the interval between points 1 and 2 have been determined according to Eq. (5).

The nonlinearity of Boltzmann plot (b) in Fig. 4 (bottom), the predictions of MS theory [1], and the data from previous experiments [5], suggest that the thermal behavior within the shock wave involves complex non-equilibrium phenomena. The results discussed below not only confirm this, but also provide a quantitative explanation.

\section{RESULTS}

\section{A. Density and temperature profiles}

The ratio between solid lines II and I in Figs. 2 and 3 accounts for the sharp density jump across the shock wave profile according to the Rankine-Hugoniot relation [14]

$$
n_{2}=\frac{(\gamma+1) M_{1}^{2}}{2+(\gamma-1) M_{1}^{2}} n_{1},
$$

as a function of the Mach number $M_{1}$ and number density $n_{1}$ at point 1 , at the onset of the shock wave. $M_{1}$ values have been estimated for $\gamma=7 / 5$ and $z_{1} / D$ according to the parametrization given elsewhere [10,13]. Though Eq. (6) strictly holds for 1D expansions of ideal gases, present results reveal that the intersection of line II with the density profile (points 2 in Fig. 2) corresponds to a discontinuity in the density gradient. Such discontinuity, better shown in Fig. 3, coincides with the complete rethermalization (nozzle exit temperature $T_{e} \approx 246 \mathrm{~K}$ ), denoting the end of the shock wave. The lag of temperatures with respect to densities within a 1D shock wave has been predicted by different theoretical approaches [1-4], and has also been demonstrated experimentally [5]. This effect is also present in 2D shock waves, as shown in Fig. 3.

Beyond point 2, however, the evolution of 2D expansions downstream the shock wave differs from the 1D case, and depends markedly on the ratio $P_{0} / P_{r}$. At point 2 rotational thermalization is complete, as shown in Fig. 3, and in more detail in Fig. 4 (spectrum $c$, and plot $c$ ). In spite of the thermalization, density profiles clearly differ between points 2 and 3 from rotational temperature profiles: Density always increase, while temperature either decrease slightly (shock waves $\mathrm{A}$ and $\mathrm{B}$ ), or remain nearly constant at about the sonic temperature (shock waves C, D, and E). Beyond that, alternating number density maxima and minima are observed at points 3 and 4 . Whether these effects are caused by secondary expansions or by the velocity inversion associated to trapped vortices [15] cannot be decided from present data.

As shown in Figs. 2 and 3, going from shock wave A to shock wave E, the discontinuity of the density gradient at the end of the shock wave (point 2) tends to fade away, indicating the lost of Rankine-Hugoniot character. This trend may be attributed to the increasing rarefaction in the gradual change from continuum to molecular flow regime, characterized by a strong reduction of the collision rate, and by a broadening of the shock wave.

\section{B. Breakdown of Boltzmann distribution}

The evolution of the population of molecular states within a shock wave is a matter of interest. MS theory [1] predicts a 
bimodal temperature distribution across the shock wave corresponding to two molar fractions, namely,

$$
x_{c}(\widetilde{z})=\left[1+\left(n_{2} / n_{1}\right) e^{4 \tilde{z} / \delta}\right]^{-1}
$$

for cold molecules, and $x_{w}=1-x_{c}$ for warm molecules; $\widetilde{z}$ $=z-z_{0}$, for $z_{0}$ the point of maximum slope of the density profile; $\left(n_{2} / n_{1}\right)$ is the density ratio across the shock wave, and $\delta$ is the shock thickness defined as $\delta=\left(n_{2}\right.$ $\left.-n_{1}\right) / \max (d n / d z)$. By cold molecules are ment those which have not yet experienced any collision with the molecules of the shock wave environment at point $\widetilde{z}$, and are still keeping the properties of the zone of silence at point 1 of Figs. 2 and 3 , namely, low temperature and high velocity. In turn, warm molecules are those which at point $\widetilde{z}$ have already experienced at least one supplementary collision and have become partially rethermalized, with the consequent reduction of speed.

A close scrutiny of the nonlinear Boltzmann plots of the rotational spectra recorded within the shock waves, as with case (b) in Fig. 4, is highly revealing: For $J \leqslant 4$ plot (b) tends asymptotically to a straight line corresponding to a temperature $T_{\text {rot }}=14 \mathrm{~K}$, while for $J \geqslant 8$ it tends asymptotically to another straight line corresponding to $T_{\text {rot }}=235 \mathrm{~K}$. These two temperatures may be related to $x_{c}$ and $x_{w}$ fractions, respectively. This confirms that MS theory [1] holds to some extent for the rotational temperature in shock waves of molecular gases [5], but proves a more complex dynamics, since part of the population corresponding to $3<J<8$ cannot be clearly assigned to $x_{c}$ nor to $x_{w}$. This suggest the existence of a third fraction $x_{s}$ not accounted for by MS theory [1]. The temperature of this fraction can only be defined as $\left\langle T_{\text {rot }}\right\rangle$ according to Eq. (5). On this basis the spectra recorded within the shock wave can univocally be reduced to the sum of three spectra, for $x_{c}, x_{w}$, and $x_{s}$ fractions, as shown in Fig. 5. Apparently, the $x_{s}$ fraction is the molecular counterpart of the scattered atoms identified in shock waves of monatomic gases [16]. Understanding the role of $x_{s}$ fraction as intermediate for the gradual conversion of $x_{c}$ into $x_{w}$ fraction across the shock wave requires a systematic experimental investigation and more elaborate shock wave models than those available today.

\section{Invariance patterns}

Data on Figs. 2 and 3 serve to obtain the temperature dependent mean free path [2]

$$
\lambda(T)=\left[\sqrt{2} \pi d_{\mathrm{ref}}^{2} n\left(T_{\mathrm{ref}} / T\right)^{\omega-1 / 2}\right]^{-1},
$$

where $d_{\text {ref }}=4.11 \AA$ is the variable soft sphere (VSS) molecular diameter of $\mathrm{N}_{2}$ at the temperature of reference $T_{\text {ref }}$ $=273 \mathrm{~K}$, and $\omega=0.74$ its viscosity index [2]. At the onset of the $2 \mathrm{D}$ shock waves, points 1 in Figs. 2 and $3, \lambda=\lambda_{1}$ may be physically identified with the homologous mean free path of 1D normal shock waves, a quantity widely employed for the normalization of lengths. The present 2D experimental results can be compared this way with 1D theoretical [2-4] and experimental results obtained by other techniques $[5,8]$. In particular, the results on shock wave $C$ very closely confirm those of Robben and Talbot [5] for a 1D shock wave of $\mathrm{N}_{2}$ at $M=12.9$.

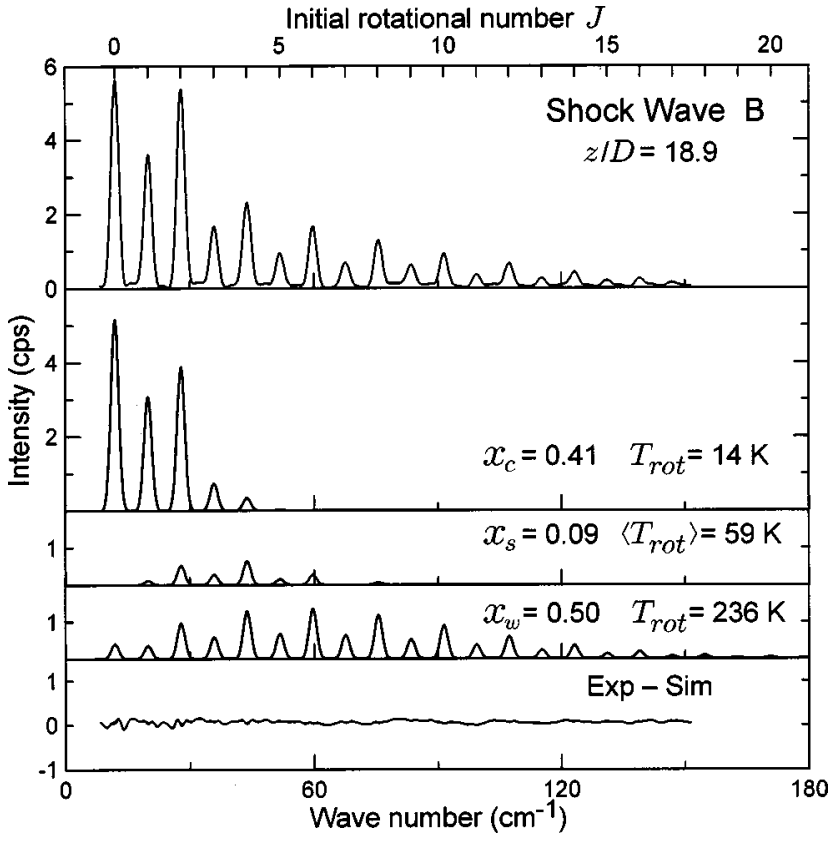

FIG. 5. Breakdown of a non-Boltzmann rotational Raman spectrum of $\mathrm{N}_{2}$, recorded within shock wave B, spectrum (b) of Fig. 4, into the simulated spectra of $x_{c}, x_{s}$, and $x_{w}$ molar fractions. Exp$\mathrm{Sim}$ is the difference between experimental and simulated spectra.

According to MS theory [1], the reduced number density profile of a 1D shock wave of a monatomic gas is given by

$$
\widetilde{n}(\widetilde{z})=\frac{n-n_{1}}{n_{2}-n_{1}}=\frac{1}{2}+\frac{1}{2} \tanh \frac{2 \widetilde{z}}{\delta} .
$$

In Fig. 6 the experimental values of $\widetilde{n}(\widetilde{z})$ are plotted versus $\widetilde{z} / \lambda_{1}$, the reduced distance expressed in terms of the mean free path at the onset of the shock wave. The solid line depicts the fit of $\widetilde{n}(\widetilde{z})$ of shock wave A to Eq. (9). These results show the invariance of the reduced density profiles for Mach

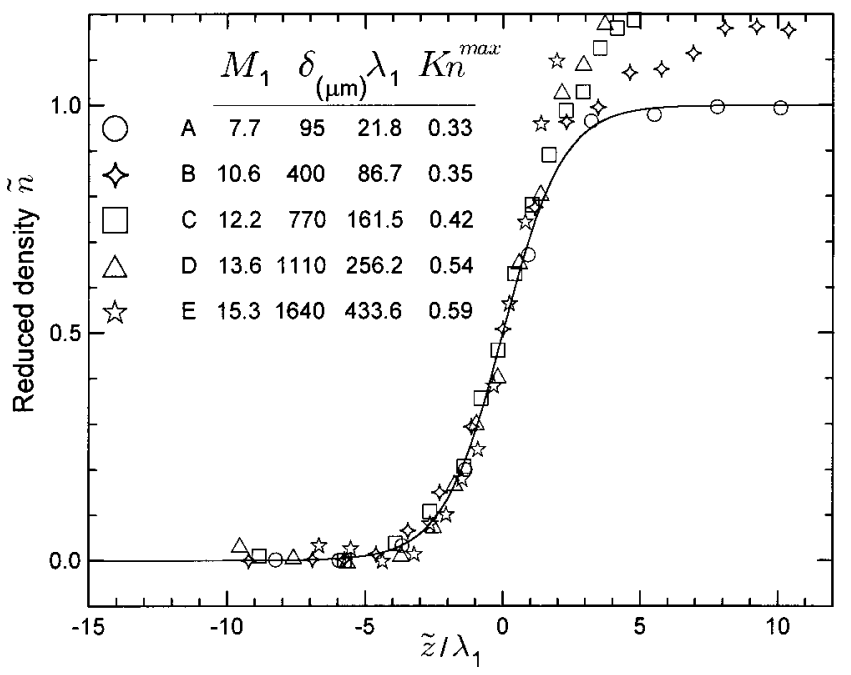

FIG. 6. Reduced density profiles of the five shock waves of $\mathrm{N}_{2}$. $M_{1}$ and $\lambda_{1}$ are Mach number and mean free path at the onset of the shock wave, $\delta$ the shock wave thickness, and $K n^{\max }=\max [(\lambda /$ $n)(d n / d z)]$, the maximum value of Knudsen number within the shock wave. 
numbers in the range $7.7<M_{1}<15.3$, and confirm the validity of Eq. (9) for most part of 2D shock waves of molecular gases, proving that their centerline density largely behaves like a $1 \mathrm{D}$ case. The increasingly larger deviations observed in the final section of the shock wave, for $\widetilde{z} / \lambda_{1}>1$ in Fig. 6 , in going from shock wave A to shock wave E, may be attributed to $2 \mathrm{D}$ scattering effects [17] in the transition from the continuum to the molecular flow regime. Such effects have not been observed in 1D shock waves [5], and are not properly accounted for by MS theory [1]. In fact, within each shock wave the local Knudsen number $K n$ reaches a maximum value, given in Fig. 6. Present maxima are well above the threshold $K n \simeq 0.2$ admitted for a flow to be tractable within the approach of continuum [2]. So, present experimental results also provide a wealth of useful data for the validation of computational codes in the poorly known transition regime from continuum to molecular flow.

\section{CONCLUSIONS}

The examples reported here show how the properties of shock waves are affected by variations of the experimental conditions, and how this problem becomes now open to accurate quantitative studies by means of Raman spectroscopy. Reference data spanning a wide range of experimental conditions can this way be produced systematically, as support for future studies involving the Boltzmann equation, and the models derived from it.

\section{ACKNOWLEDGMENT}

The Spanish SEUID is acknowledged for financial support (research project PB97-1203).
[1] H. M. Mott-Smith, Phys. Rev. 82, 885 (1951).

[2] G. A. Bird, Molecular Gas Dynamics and the Direct Simulation of Gas Flows (Clarendon Press, Oxford, 1994).

[3] K. Koura, Phys. Fluids 9, 3543 (1997).

[4] I. A. Chirokov, T. G. Elizarova, and J. C. Lengrand, in Proceedings of the 21st International Symposium on Rarefield Gas Dynamics, edited by R. Brun (Cepadues Editions, Toulouse, 1999), Vol. 1, p. 175.

[5] F. Robben and L. Talbot, Phys. Fluids 9, 633 (1966); 9, 653 (1966).

[6] E. P. Muntz, Phys. Fluids 11, 64 (1968).

[7] R. B. Smith, Phys. Fluids 15, 1010 (1972).

[8] H. Alsmeyer, J. Fluid Mech. 74, 497 (1976).

[9] S. Montero, B. Maté, G. Tejeda, J. M. Fernández, and A. Ramos, in Atomic and Molecular Beams, edited by R. Campargue (Springer-Verlag, Berlin, in press).

[10] G. Tejeda, B. Maté, J. M. Fernández-Sánchez, and S. Montero,
Phys. Rev. Lett. 76, 34 (1996).

[11] G. Tejeda, J. M. Fernández-Sánchez, and S. Montero, Appl. Spectrosc. 51, 265 (1997).

[12] H. Ashkenas and F. S. Sherman, in Proceedings of the Fourth International Symposium on Rarefield Gas Dynamics, edited by J. H. de Leeuw (Academic, New York, 1966), Vol. 2, p. 84.

[13] D. R. Miller, in Atomic and Molecular Beams Methods, edited by G. Scoles (Oxford University Press, New York, 1988), Vol. I.

[14] M. J. Zucrow and J. D. Hoffman, Gas Dynamics (Wiley, New York, 1976), Vol. I.

[15] C. L. Chen, S. R. Chakravarthy, and C. M. Hung, AIAA J. 32, 1836 (1994).

[16] G. Pham-Van-Diep, D. Erwin, and E. P. Muntz, Science 245, 624 (1989).

[17] E. P. Muntz, B. B. Hamel, and B. L. Maguire, AIAA J. 8, 1651 (1970). 